\title{
COMPASS results on weighted Sivers asymmetry in SIDIS
}

\author{
Anna Martin* \\ Trieste University and INFN \\ E-mail: anna.martin@ts.infn.it

\section{on behalf of the COMPASS Collaboration}

The transverse momentum weighted asymmetries have been proposed since a long time to access the transverse momentum dependent parton distributions. The COMPASS Collaboration has recently measured the weighted Sivers asymmetries in deep inelastic lepton scattering off transversly polarised protons. The results are in agreement with the expectations based on the previous measurements of the standard Sivers asymmetries. They are briefly described here, together with a new point-by-point extraction of the first moment of the Sivers function.

23rd International Spin Physics Symposium - SPIN2018 -

10-14 September, 2018

Ferrara, Italy

\footnotetext{
${ }^{*}$ Speaker.
} 
The Sivers distribution function $f_{1 T}^{\perp q}[1,2]$ is one of the most studied among the eight transversemomentum-dependent distributions (TMDs) which are required for a complete description of the nucleon structure at leading twist. The TMDs depend on the parton light-cone momentum fraction $x$ and on the transverse momentum $k_{T}$ of the parton with respect to the nucleon direction. Upon integration over the transverse momentum, only three of these distributions survive: the number density, the helicity and the transversity distributions.

For a transversely polarised nucleon, the Sivers function describes the correlation between the transverse momentum $k_{T}$ of an unpolarised quark of flavour $q$ and the polarisation of the nucleon. It can be accessed in different channels, like semi-inclusive measurements of lepton deep-inelastic scattering (SIDIS) and Drell-Yan processes when transversely polarised targets are used. Because of its "T-odd" nature, it is expected to have opposite sign in SIDIS and Drell-Yan processes and the test of this property is regarded as a fundamental test of the present theoretical framework.

In SIDIS off transversely polarised nucleons, the Sivers function is responsible for a $\sin \Phi_{S}$ modulation in the cross-section, which schematically can be written as:

$$
\mathrm{d} \sigma=\mathrm{d} \sigma_{U}+S_{T} \mathrm{~d} \sigma_{S} \sin \Phi_{\mathrm{Siv}}
$$

where $S_{T}$ is the target nucleon polarisation, $\mathrm{d} \sigma_{U}$ is the spin-independent cross-section, $\mathrm{d} \sigma_{S}$ is the spin-dependent part containing the Sivers function, and the other spin-dependent terms have been omitted. The angle $\Phi_{S}=\phi_{h}-\phi_{S}$ is the difference between the azimuthal angle of the produced hadron and that of the target nucleon spin, in a reference system in which the $\mathrm{z}$ axis is defined by the virtual photon direction. The Sivers function contribute to $\mathrm{d} \sigma_{S}$ and can thus be accessed by measuring the amplitude of the $\sin \Phi_{\text {Siv }}$ modulation, i.e. by weighting the events with $\sin \Phi_{S}$ in order to project out from the data the Sivers spin asymmetry. Adding into the weight a power $P_{T}$ (the component of the momentum of the produced hadron transverse to the virtual photon direction) allows to extract from the spin asymmetry in a model independent way the first transverse momenta of $f_{1 T}^{\perp(1) q}$ of the Sivers functions:

$$
f_{1 T}^{\perp(1) q}(x)=\int d^{2} k_{T} \frac{k_{T}^{2}}{2 M^{2}} f_{1 T}^{\perp q}\left(x, k_{T}^{2}\right) .
$$

The same method can be used to access the other TMDs which contribute to the SIDIS [3,4] and to the Drell-Yan $[5,6]$ cross-sections ${ }^{1}$.

For some reasons, the $P_{T}$ weighted Sivers asymmetries were never measured (till now only preliminary results were produced by the HERMES Collaboration [8]). The Sivers asymmetries measured in so far by the HERMES [9] and the COMPASS [10] Collaborations are not weighted with $P_{T}$ and can be written as:

$$
A_{\text {Siv }}=2 \frac{\int \mathrm{d} \Phi_{\text {Siv }} \mathrm{d} \phi_{h} \sin \Phi_{\text {Siv }} \mathrm{d} \sigma}{\int \mathrm{d} \Phi_{\text {Siv }} \mathrm{d} \phi_{h} \mathrm{~d} \sigma}
$$

Different analyses of the existing data allowed for extractions of the Sivers functions and of their first transverse moments [11, 12, 13, 14, 15]. Because of the convolution over transverse momenta

\footnotetext{
${ }^{1}$ Preliminary results for the weighted Sivers asymmetry in Drell-Yan have been recently produced by the COMPASS Collaboration [7].
} 
which appears at the numerator of eq. (3), however, some model for the dependence on the transverse momenta of the Sivers function $f_{1 T}^{\perp}$ and of the fragmentation function $D_{1}$, had to be used. The most widely used model is the Gaussian ansatz, namely the assumption that the distribution of transverse momenta both in the Sivers and in the fragmentation functions has a Gaussian shape. With this assumption the convolution can be carried out and the unweighted Sivers asymmetry can be explicitly written in terms of $f_{1 T}^{\perp(1) q}[4,5]$ :

$$
A_{\mathrm{Siv}, \mathrm{G}}(x, z)=\frac{a_{\mathrm{G}} \sum_{q} e_{q}^{2} x f_{1 T}^{\perp(1) q}(x) z D_{1}^{q}(z)}{\sum_{q} e_{q}^{2} x f_{1}^{q}(x) D_{1}^{q}(z)} .
$$

Here

$$
a_{\mathrm{G}}=\frac{\sqrt{\pi} M}{\sqrt{\left\langle p_{T}^{2}\right\rangle+z^{2}\left\langle k_{T}^{2}\right\rangle_{S}}} \simeq \frac{\pi M}{2\left\langle P_{T}\right\rangle},
$$

where $\left\langle p_{T}^{2}\right\rangle$ and $\left\langle k_{T}^{2}\right\rangle_{S}$ are the Gaussian widths of the fragmentation functions and of the Sivers functions assumed to be flavor independent, ans $z$ if fraction of the available energy carried by the hadron.

In spite of the successfully description of the existing data, which demonstrates that within the present accuracy of the data the Gaussian ansatz is reliable, the $P_{T}$ weighted spin asymmetries are still attractive being a novel and complementary method to access the Sivers function.

Very recently ${ }^{2}$ COMPASS has performed a new analysis of the existing data and has measured the weighted spin asymmetries

$$
A_{\text {Siv }}^{W}=\frac{\int \mathrm{d} \Phi_{\text {Siv }} \sin \Phi_{\text {Siv }} \int \mathrm{d}^{2} P_{T} W \mathrm{~d} \sigma}{\int \mathrm{d} \Phi_{\text {Siv }} \int \mathrm{d}^{2} P_{T} \mathrm{~d} \sigma}
$$

where $W$ is the $P_{T}$ weight taken to be either $W=w=P_{T} / z M$ or $W=w^{\prime}=P_{T} / M$. With both weights, the convolution can be carried out without assumptions on the transverse momenta distributions, and the asymmetries $A_{\mathrm{Siv}}^{W}$ can be written as:

$$
\begin{gathered}
A_{\text {Siv }}^{w}(x, z)=2 \frac{\sum_{q} e_{q}^{2} x f_{1 T}^{\perp(1) q}(x) D_{1}^{q}(z)}{\sum_{q} e_{q}^{2} x f_{1}^{q}(x) D_{1}^{q}(z)}, \\
A_{\text {Siv }}^{w^{\prime}}(x, z)=2 \frac{\sum_{q} e_{q}^{2} x f_{1 T}^{\perp(1) q}(x) z D_{1}^{q}(z)}{\sum_{q} e_{q}^{2} x f_{1}^{q}(x) D_{1}^{q}(z)} .
\end{gathered}
$$

When integrating over $z, A_{\text {Siv }}^{w}$ has a particularly simple expression, and, assuming u-quark dominance for the production of positive hadrons on a proton target, it directly gives $f_{1 T}^{\perp(1) u}(x) / f_{1}^{u}(x)$. On the other hand, $A_{\text {Siv }}^{w^{\prime}}$ is more similar to unweighted asymmetry in the Gaussian model, thus the measurements of both the asymmetries are interesting.

The data used in this analysis were collected in 2010 by scattering a $160 \mathrm{GeV} \mu^{+}$beam off a transversely polarised proton $\left(\mathrm{NH}_{3}\right)$ target. The event and hadron selections are the same used in the previous analysis [10]. In order to ensure the DIS regime events with photon virtuality $Q^{2}>1(\mathrm{GeV} / c)^{2}$, fractional energy of the virtual photon $0.1<y<0.9$, and mass of the hadronic

\footnotetext{
${ }^{2}$ The results have just been published [16].
} 

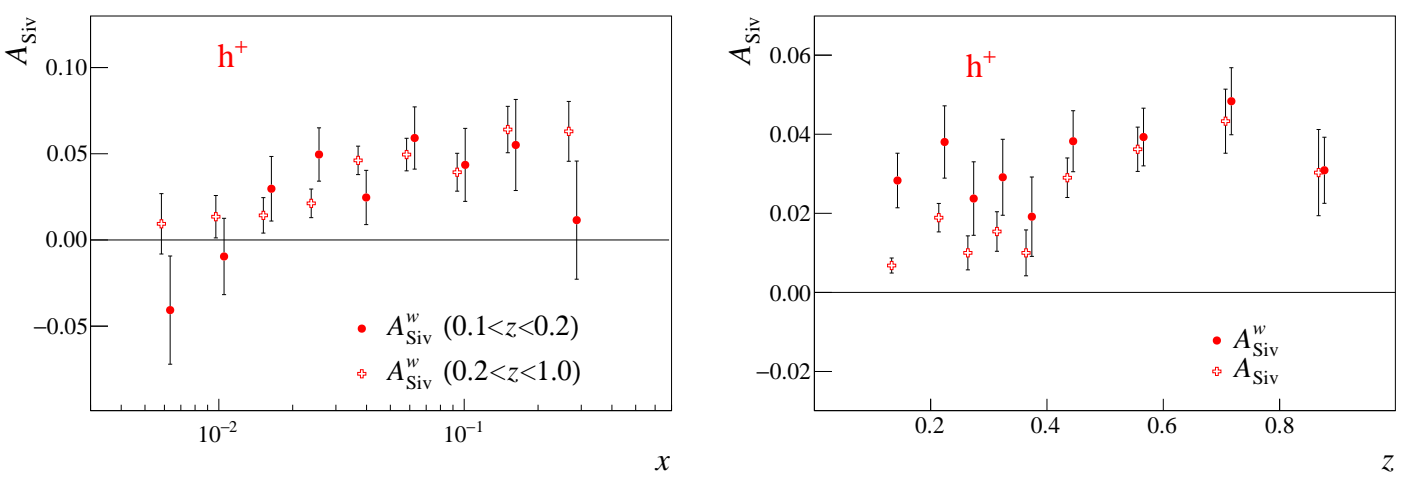

Figure 1: Weighted asymmetries for positive hadrons. Left: $A_{\text {Siv }}^{w}$ vs. $x$ measured in the range $0.1<z<0.2$ (closed points) and in the range $z>0.2$ (open points). Right: $A_{\text {Siv }}^{w}$ vs. $z$ (closed points) compared with the unweighted Sivers asymmetries from Ref. [10].

final-state system $W>5 \mathrm{GeV} / c^{2}$ are selected. The charged hadrons (pions in about the $70 \%$ of the cases) are required to have $P_{T} \geq 0.1 \mathrm{GeV} / c$ and $z>0.2$ or $0.1<z<0.2$. The double ratio method already used in previous analyses to measure the spin asymmetries has been slightly modified [16] in order to weight with $w$ or $w^{\prime}$ only the spin-dependent part of the cross-section. Several tests have been performed to access systematic effects. In particular alternative estimators were used, getting essentially identical results. The effects of the acceptance and cuts in $P_{T}$ have also been investigated finding them to be negligible.

The weighted asymmetries have been extracted separately for positive and negative hadrons as a function of $x$ or $z$. In these proceeding only some of the results are included. More results and all tables can be found in Ref. [16].

The weighted Sivers asymmetries $A_{\text {Siv }}^{w}$ for positive hadrons are shown in Fig. 1. In the left plot the asymmetries are shown as a function of $x$ for the range $z>0.2$ (open crosses), which is the "standard" $z$ range for COMPASS results, and for the range $0.1<z<0.2$ (closed points). The two sets of points are compatible, namely the positive-hadron asymmetries are basically unchanged at low $z$, as expected if u-quark dominance for positive hadrons produced on a proton target and factorisation hold in the COMPASS phase space. With these assumptions, one has $A_{\text {Siv }}^{w}(x) \simeq 2 f_{1 T}^{\perp(1) u}(x) / f_{1}^{u}(x)$ and the values of the weighted Sivers asymmetry shown in the figure are a direct measurement of transverse moment of the Sivers function. Also, the $x$ dependence is the same of that of the unweighted asymmetries, and the ratio of the two asymmetries is almost constant and compatible with the mean values of the weight. In the right plot of Fig. 1 the measured values of $A_{\text {Siv }}^{w}$ (closed circles) as function of $z$ are compare with those of $A_{\text {Siv }}$ [10] (open crosses). As expected, the weighted asymmetry is almost constant, at variance with $A_{\text {Siv }}$.

The left plot of Fig. 2 shows the weighted asymmetry for negative hadrons in the nine $x$ bins. In the range $z>0.2$ the values (open crosses) are compatible with zero (apart from the highest $x$ point), as much as the unweighted asymmetry, in agreement with a cancellation between $\mathrm{u}$ - and d-quark contributions. For $0.1<z<0.2$ the values of $A_{\text {Siv }}^{w}$ become larger than zero at $x>0.01$ and close to those of the asymmetry for positive hadrons. This is qualitatively expected since at low $z$ the favored and unfavored fragmentation functions become similar and thus the u-quark 

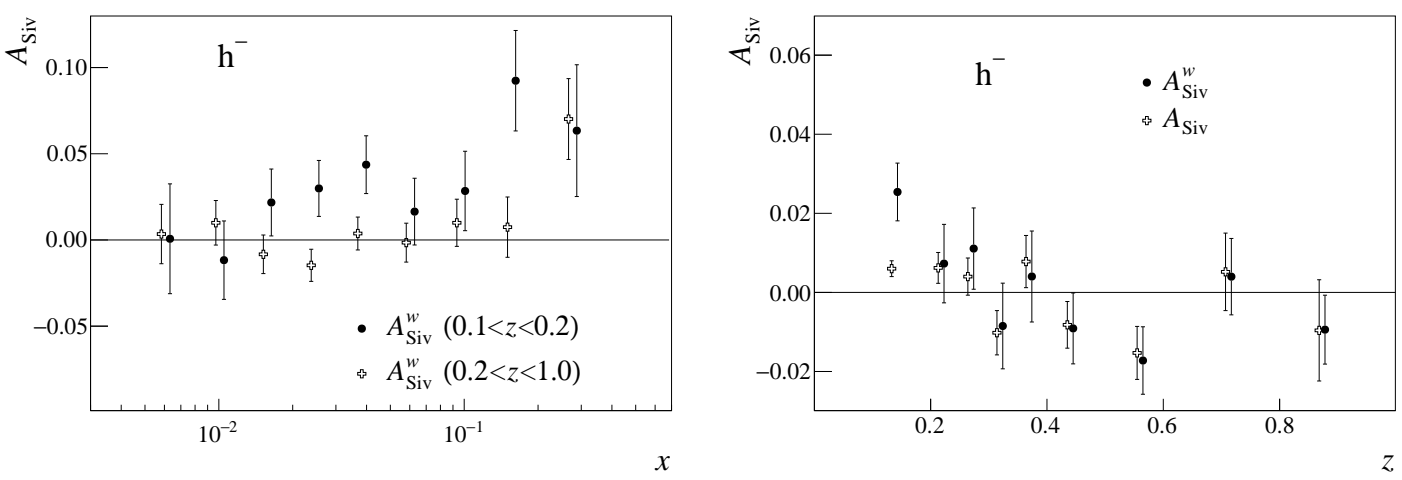

Figure 2: Weighted asymmetries for negative hadrons. Left: $A_{\text {Siv }}^{w}$ vs. $x$ measured in the range $0.1<z<0.2$ (closed points) and in the range $z>0.2$ (open points). Right: $A_{\text {Siv }}^{w}$ vs. $z$ (closed points) compared with the unweighted Sivers asymmetries from Ref. [10].

contribution dominates. The $z$ dependence can be better seen in the right plot of the same figure where the $x$ integrated values of $A_{\text {Siv }}^{w}$ are shown as open crosses. The closed circles show for comparison the unweighted asymmetries. Since for negative hadrons the d-quark contribution is relevant, in particular at lager $z$, deuteron data are needed to learn more. Unfortunately no significant measurement of the weighted Sivers asymmetry can be done using the existing deuteron data, which have very limited statistics and were collected at the beginning of COMPASS with a reduced acceptance spectrometer.

The weighted Sivers asymmetries $A_{\text {Siv }}^{w}$ for positive and negative hadrons measured at $z>0.2$ have been used for a point-by-point extraction of the first transverse moments $f_{1 T}^{\perp(1) u_{v}}$ and $f_{1 T}^{\perp(1) d_{v}}$ following the procedure of Ref. [15]. In that work the unweighted Sivers asymmetries for positive and negative pions and kaons measured on proton [17] and deuteron [18] were used. In the present analysis, since the weighted asymmetries on deuteron are not available, we had to neglect the seaquark Sivers distributions, which anyhow was found to be compatible with zero in the analysis of Ref. [15]. The new results are shown in Fig. 3 as closed red and open black circles for the $u$ and $\mathrm{d}$ quarks respectively. As in previous extractions, $f_{1 T}^{\perp(1) u_{v}}$ is clearly positive for $x>0.01$ while $f_{1 T}^{\perp(1) d_{v}}$ has negative values. The error bars (statistical uncertainties only) are much smaller for the u quark, as expected. The squares in Fig. 3 show the results of Ref. [15]. The agreement is very good for the u quark, and the new values of $f_{1 T}^{\perp(1) d_{v}}$ are lower than the previous ones. By repeating the previous analysis, however, we have checked that the difference is mainly due to the use in Ref. [15] of the deuteron data and to the extraction of the sea-quark Sivers function rather than to the use of the unweighted asymmetries and of the Gaussian ansatz.

COMPASS has also measured the Sivers asymmetries weighted with $w^{\prime}=P_{T} / M$ which are expected to have a $z$ dependence similar to that of the unweighted asymmetries $A_{\text {Siv }}$. The asymmetries $A_{\text {Siv }}^{w^{\prime}}$ have been measured in bins of $x$ for the two $z$ ranges, and in bins of $z$ [16]. The ratios $A_{\text {Siv }}^{w^{\prime}} / A_{\text {Siv }}$ for positive hadrons are shown in Fig. 4 . As can be seen the trend is quite similar a part from a possible scale factor.

To summarise, the COMPASS Collaboration has measured the weighted Sivers asymmetries in SIDIS off transversely polarised protons, demonstrating the feasibility of the measurement. These 


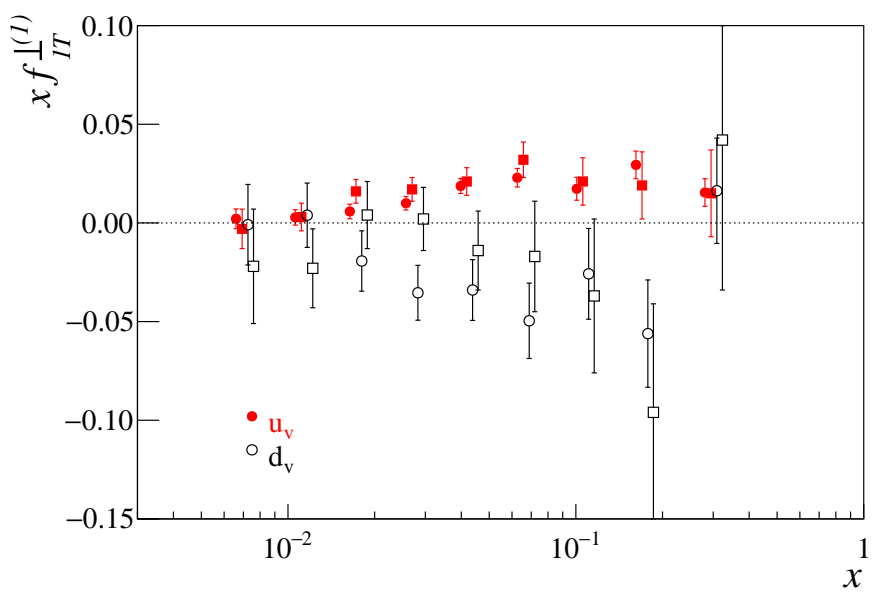

Figure 3: Comparison of the values of the first moment of the Sivers function for $u$ (closed red dots) and $d$ (open black dots) quarks from the $P_{T} / z M$-weighted Sivers asymmetries for charged hadrons with $z>0.2$, and the corresponding values obtained in Ref. [15] from the unweighted pion Sivers asymmetries measured by COMPASS on deuteron and proton (closed red and open black squares, respectively).
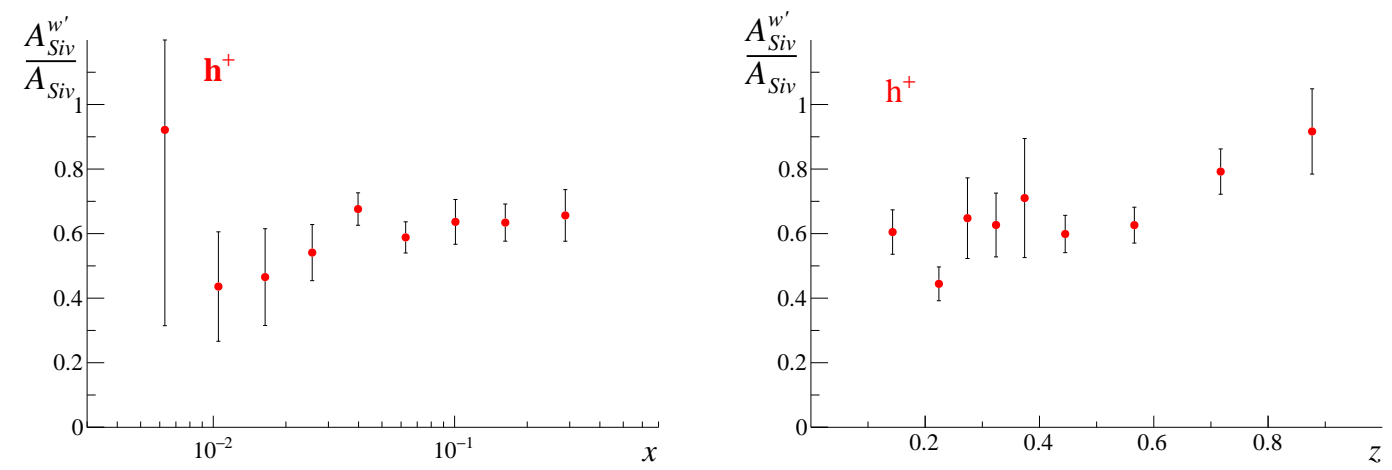

Figure 4: Ratio $A_{\text {Siv }}^{w^{\prime}} / A_{\text {Siv }}$ for positive hadrons as a function of $x$ for $z>0.2$ (left) and as function of $z$ (right). The black points are the mean values of the weight $4\left\langle P_{T} / M\right\rangle$.

asymmetries allow for a complementary access to the Sivers function with respect to the standard ones, since no assumption on the transverse momenta dependence of the TMD distributions and fragmentation functions is required. Looking at the $x$ and $z$ dependences of these novel asymmetries no indication for contamination from target fragmentation has been observed. The new data have also been used to extract the first transverse moments of the Sivers function finding results in substantial agreement with the previous extractions based on the standard Sivers asymmetries and the Gaussian ansatz. More information will be obtained when the new deuteron data will be collected in 2021. They will allow for a more detailed comparison with the standard Sivers asymmetries and for a much more accurate determination of the d-quark distribution.

\section{References}

[1] D. W. Sivers, Phys. Rev. D 41 (1990) 83. 
[2] D. W. Sivers, Phys. Rev. D 43 (1991) 261.

[3] A. M. Kotzinian and P. J. Mulders, Phys. Rev. D 54 (1996) 1229.

[4] D. Boer and P. J. Mulders, Phys. Rev. D 57 (1998) 5780.

[5] A. V. Efremov, K. Goeke, S. Menzel, A. Metz and P. Schweitzer, Phys. Lett. B 612 (2005) 233.

[6] A. Sissakian, O. Shevchenko, A. Nagaytsev, O. Denisov and O. Ivanov, Eur. Phys. J. C 46 (2006) 147.

[7] J. Matoušek [COMPASS Collaboration], J. Phys. Conf. Ser. 938 (2017) no.1, 012012, and these Proceedings.

[8] I. M. Gregor [HERMES Collaboration], Acta Phys. Polon. B 36 (2005) 209.

[9] A. Airapetian et al. [HERMES Collaboration], Phys. Rev. Lett. 103 (2009) 152002.

[10] C. Adolph et al. [COMPASS Collaboration], Phys. Lett. B 717 (2012) 383.

[11] M. Anselmino et al., "Comparing extractions of Sivers functions," Proceedings of Transversity 2005, 7-10 Sep 2005, Como, Italy, World Scientific, 2006, hep-ph/0511017.

[12] M. Anselmino, M. Boglione and S. Melis, Phys. Rev. D 86 (2012) 014028.

[13] P. Sun and F. Yuan, Phys. Rev. D 88 (2013) 034016.

[14] M. G. Echevarria, A. Idilbi, Z. B. Kang and I. Vitev, Phys. Rev. D 89 (2014) 074013.

[15] A. Martin, F. Bradamante and V. Barone, Phys. Rev. D 95 (2017) no.9, 094024.

[16] M. G. Alexeev et al. [COMPASS Collaboration], Nucl. Phys. B 940 (2019) 34.

[17] C. Adolph et al. [COMPASS Collaboration], Phys. Lett. B 744 (2015) 250.

[18] M. Alekseev et al. [COMPASS Collaboration], Phys. Lett. B 673 (2009) 127. 